\section{Acute pediatric cerebellitis and mutism. Case report and review of the literature}

\author{
CLAUDIA FANTACCI • GIULIA BERSANI • PIERO VALENTINI • \\ FILOMENA PIERRI • PAOLO MARIOTTTI • DANIELE SERRANTI • \\ ANTONIO CHIARETTI
}

ANTONIO CHIARETTI $(\square) \cdot$ CLAUDIA FANTACCI • GIULIA BERSANI • PIERO VALENTINI • FILOMENA PIERRI • DANIELE SERRANTI

Department of Pediatrics Catholic University Medical School of Rome, Italy Largo A. Gemelli 8 00168 Rome, Italy Phone: +39-06-30154690

Fax:: +39-06-3383211

E-mail: achiaretti@yahoo.it

PAOLO MARIOTTTI

Unit of Pediatric Neurology, Catholic University Medical School of Rome, Italy

\begin{abstract}
Acute cerebellitis $(A C)$ is a rare syndrome characterized by an inflammatory involvement of the cerebellum which can complicate infections or vaccinations. AC can be a life-threatening condition, presenting with speech disorders including longlasting mutism, usually followed by dysarthria. Clinical examination, laboratory findings and Magnetic Resonance Imaging are essential for establishing an early diagnosis and initiating prompt and adequate treatment, thereby reducing morbidity and mortality. We report a case of a child with AC complicated by mutism and we present a review of the literature of pediatric cases with this condition.
\end{abstract}

Key words: cerebellitis, mutism, dysarthria, childhood

\section{Introduction}

Acute cerebellitis (AC) is an uncommon syndrome characterized by inflammatory involvement of the cerebeIlum, which can complicate infections or vaccinations. Its main manifestations are ataxia, a wide-based gait, dysmetria, tremor, fever, headache, nystagmus and speech disorders, including mutism. (1) Cerebellar mutism is a complete but transient loss of speech, usually followed by dysarthria, caused by direct lesions of the cerebellar vermis or hemispheres. (2) It is commonly reported as a complication after posterior fossa neurosurgery, while it is less frequent in cerebellar inflammation. (3) The neuroimaging gold standard for the diagnosis of AC is Magnetic Resonance
Imaging (MRI), usually demonstrating altered signal intensity, swelling and abnormal diffusion weighted imaging of the cerebellum and surrounding areas. (4) Antibiotic, antiviral, antiedema and/ or corticosteroid drugs are useful for treating $\mathrm{AC}$, while rarely external ventricular drainage is necessary to reduce intracranial hypertension. (1) AC usually has a rapid onset and is self-limiting, but sometimes can be life-threatening. (5) Its symptoms and complications, especially mutism, can last for severa months. We report a case of a child with AC complicated by mutism, and we present a review of the literature of pediatric cases with this condition.

\section{Case report}

A 6-year-old previously healthy girl presented with fever, diarrhea, seizures, neck stiffness, upward eye deviation and drowsiness. She was admitted to our Pediatric intensive care unit (PICU) (day 0), where she required two days of mechanical ventilation to control persisting seizures and respiratory complications. Cerebrospinal fluid (CSF) examination revealed 900 cells $/ \mathrm{mmc}$, protein $164 \mathrm{mg} / \mathrm{dl}$ and glucose $54 \mathrm{mg} /$ dl. C-Reactive-Protein was increased (60.38 mg/L; normal value $<5$ mg/L). Blood cell count and blood chemistry were normal. Suspecting viral encephalitis, therapy with Acyclovir was started. Electroencephalogram (EEG) showed beta and delta activity in medio-posterior brain regions, with right prevalence. Serological tests for cytomegalovirus, varicella-z virus, herpes simplex virus 6 , adenovirus, rubella and mumps demonstrated past infections; bacterial and mycotic blood cultures and serology for toxoplasma gondii, coxsackie virus, 


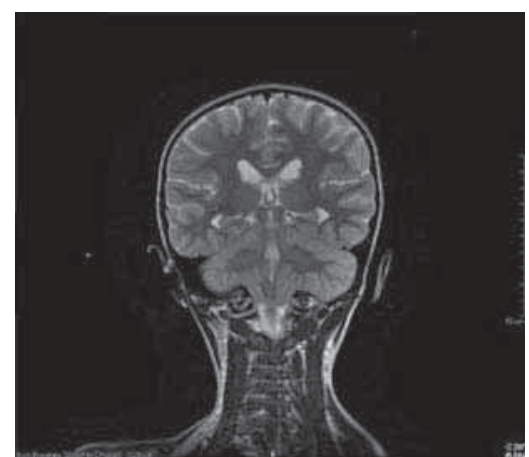

Figure 1A.

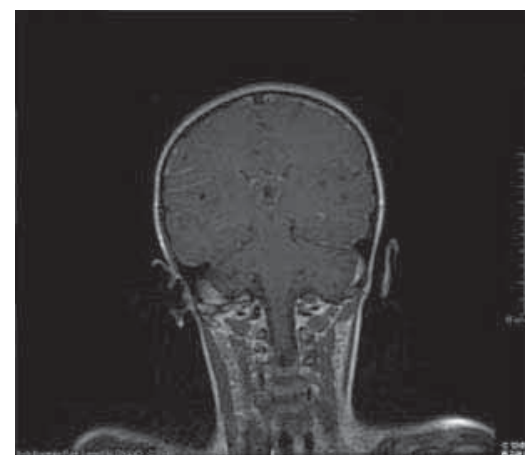

Figure 1B.

Figure 1. MRI of the child with acute cerebellitis. Figure 1A shows herniation of the cerebellar tonsils encroaching upon the foramen magnum at the moment of diagnosis. Figure 1B reveals the regression of the cerebeIlar tonsillar dislocation and minimal dilatation of the fourth ventricle, six months after the diagnosis.

Epstein-Barr virus, herpes simplex virus type 1 and 2 and Borrelia burgdoferi were negative, while IgA and IgM antiEchovirus were detected. Pharyngeal and fecal cultures were negative. The first MRI, performed using FSE, GRE, FLAIR and DWI sequences, showed the herniation of cerebellar tonsils through the foramen magnum ( $6 \mathrm{~mm}$ below the Chamberlain line) (figure 1A). On day 8 the child was awake, being able to perform simple commands but she demonstrated mutism. She could not walk and perform fine movements. Four days later, she started walking with ataxia. On day 21 she presented with dysarthric and fragmented speech and a new EEG revealed diffuse, slow basic activity. A second MRI, performed on day 30 , did not demonstrate significant differences from the first one. With persisting symptoms, a single photon emission tomography (SPET) was performed, showing the absence of cerebral and cerebellar regional deficit of perfusion. The child was discharged on day 34: her motor functions, walking and speech improved, she restarted drawing simple subjects but she still presented with dysarthria and equilibrium disturbances. At clinical follow-up, 6 months after the onset of symptoms, she was able to formulate sentences, although she still spoke slowly. Neurological examination was normal except for slight nystagmus laterally. MRI, performed at the same time, revealed the regression of cerebellar tonsillar dislocation; in addition, it showed slight widening of perifolial liquoral spaces and a minimal dilatation of the fourth ventricle (figure 1B). This study was approved by the Institutional Review Board (IRB) and written informed consent was signed by the child's parents.

\section{Discussion}

$A C$ is an inflammatory syndrome which involves cerebellar structures usually bilaterally and symmetrically, as a consequence of viral infections or vaccinations. The main symptoms and signs are related to cerebellum dysfunction, associated with abnormal cerebrospinal fluid findings and an abnormal MRI. (1) AC is most frequent in childhood and is usually self-limiting; though sometimes, it can be a life-threatening event, leading to serious complications including death. (5) Its etiology is various, usually viral (herpes virus, rubella, Coxsackie virus); more rarely B. burgdoferi, Coxiella burnetii and Mycoplasma pneumonia have been involved. (5) MRI of the brain is the radiologic investigation of choice for the detection of inflammatory disorders of the cerebellum, and it is also useful for their follow-up. (4) Cranial Axial Computed Tomography (CAT) has been demonstrated to be often unrevealing. (4) Brain SPECT may be useful to better assess the neurological deficits as it shows marked reduction in cerebral perfusion. (6) Our case reveals some interesting characteristics because the child suffered from an episode of acute cerebellitis, likely consequent to an Echovirus infection, complicated by persistent mutism but without important alterations of SPECT findings. Only MRI showed herniation of the cerebellar tonsils, confirming the leading role of MRI in diagnosing AC. In the literature, we found 17 cases of pediatric acute cerebellitis presenting with mutism (table 1). (2,3,7-10) These cases concerned 12 girls and 5 boys with age range between 2 and 7 years. Mutism lasted between 3 days and 5 months (mean: 21 days) and was followed by dysarthria, with slow and difficult language recovery. The etiology was unknown in 13 cases (76.5\%) and the involved agent was always Rotavirus, detected only in stool samples. All children underwent MRI examination, revealing, more frequently, hyperintense areas and swelling of the cerebellum in T2-weighted sequences. Only in one child was tonsillar herniation detected, as happened in our case. No other patients underwent a SPECT examination. In regard to treatment, only one child required external ventricular drainage due to intracranial hypertension, while everyone else received antibiotics, antiviral, antiedema and/or corticosteroid drugs. Follow-up reported in the literature differed according to timing and findings. Clinically, every child improved in his/her ability to walk and speak. Speech disorders often persisted, lasting several months, and perfectly fluent speech was not found at clinical follow-up. Radiological follow-up showed cerebellar atrophy in 16 children (94\%).

In conclusion, we can state that $A C$ is a dangerous syndrome, often complicated by long-lasting mutism affecting children's quality of life. Clinical examination, CSF findings and MRI are essential for establishing an early diagnosis and initiating prompt treatment to reduce the morbidity and mortality associated with this condition. 


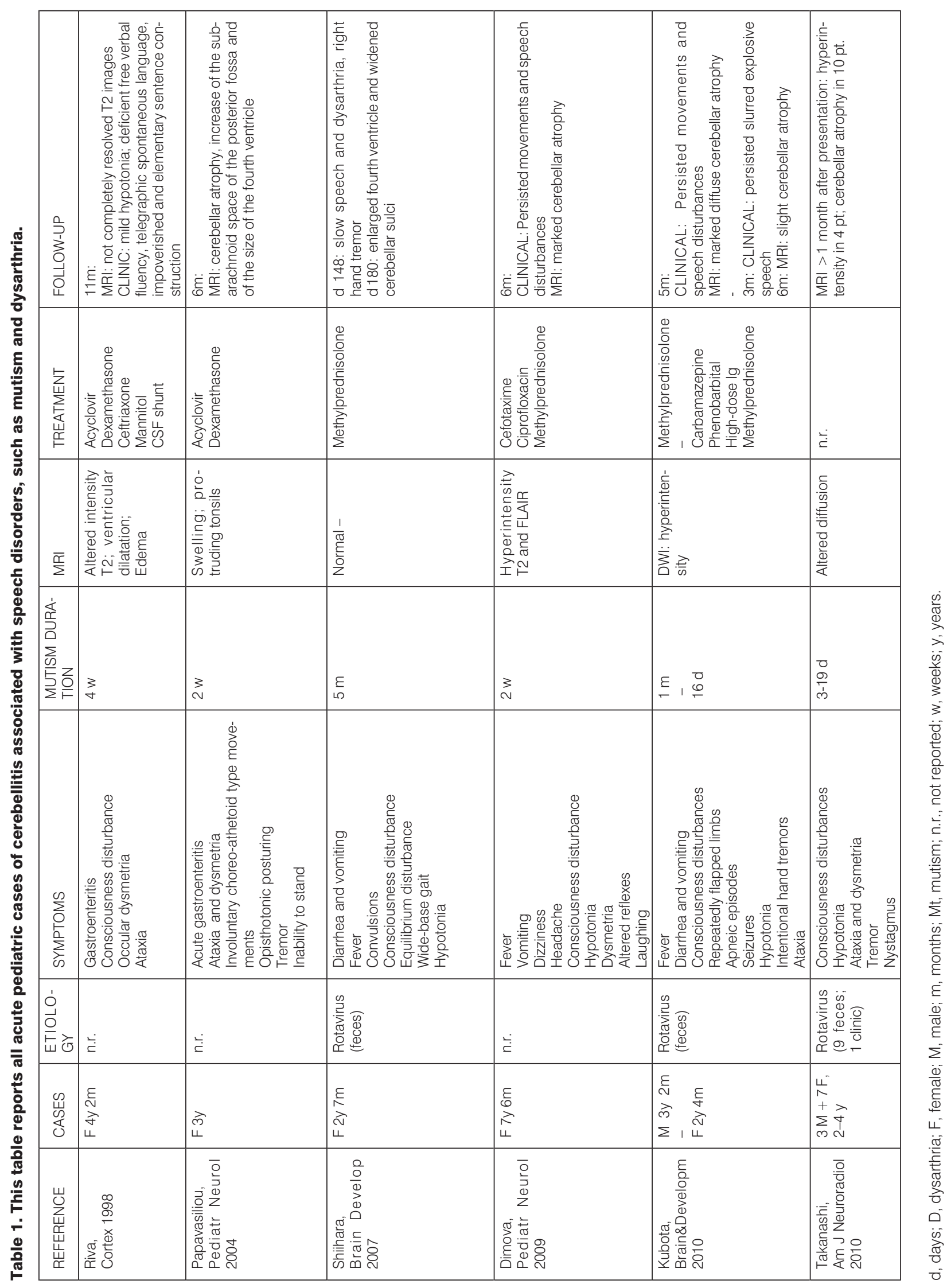




\section{REFERENCES}

1. Sawaishi Y, Takada G. Acute cerebellitis. Cerebellum 2002;1:223-8.

2. Riva D. the cerebellar contribution to language and sequential functions: evidence from a child with cerebellitis. Cortex 1998;34:279-87.

3. Kubota T, Suzuki T, Kitase Y, Kidokoro H, Miyajima Y, Ogawa A, Natsume J, Okumura A. Chronological diffusion-weighted imaging changes and mutism in the course of rotavirus-associated acute cerebellitis/cerebellopathy concurrent with encephalitis/encephalopathy. Brain Dev 2011;33:21-7.

4. De Bruecker Y, Claus F, Demaerel P, Ballaux F, Sciot R, Lagae L, Buyse G, Wilms G. MRI findings in acute cerebellitis. Eur Radiol 2004;14:781-3.

5. Levy El, Harris AE, Omalu BI, Hamilton RL, Branstetter BF 4th, Pollack IF. Sudden death from fulminant acute cerebellitis. Pediatr Neurosurg 2001;35:24-8.

6. San Pedro EC, Mountz JM, Liu HG, Deutsch G. Postinfectious cerebellitis: clinical significance of Tc-99m HMPAO brain SPECT compared with MRI. Clin Nucl Med 1998;23:212-6.

7. Papavasiliou AS, Kotsalis C, Trakadas S. Transient cerebellar mutism in the course of acute cerebellitis. Pediatr Neurol 2004;30:71-4.

8. Shiihara T, Watanabe M, Honma A, Kato M, Morita Y, Ichiyama T, Maruyama K. Rotavirus associated acute encephalitis/encephalopathy and concurrent cerebellitis: report of two cases. Brain Dev 2007;29:670-3.

9. Dimova PS, Bojinova VS, Milanov IG. Transient mutism and pathologic laughter in the course of cerebellitis. Pediatr Neurol 2009;41:49-52.

10. Takanashi J, Miyamoto T, Ando N, Kubota T, Oka M, Kato Z, Hamano S, Hirabayashi S, Kikuchi M, Barkovich AJ. Clinical and radiological features of rotavirus cerebellitis. Am J Neuroradiol 2010;31:1591-5. 